Check for updates

Cite this: Chem. Commun., 2020, 56, 13776

Received 30th September 2020 , Accepted 13th October 2020

DOI: 10.1039/d0cc06552h

rsc.li/chemcomm

\section{Organic single crystals of cyano-substituted $p$-phenylene vinylene derivatives as transistors with low surface trap density $\dagger$}

\author{
Jian Deng, (D) $\ddagger$ Yuejuan Wan, (D) $\ddagger$ Chang Cai, (D) Cheng Gu (D) * and \\ Yuguang $\mathrm{Ma}$ (D) *
}

\begin{abstract}
We obtained two high-quality lamellar crystals of cayno-substituted $p$-phenylene vinylene derivatives and fabricated their transistors. The transistors demonstrated hysteresis-free, low subthreshold swing values of 0.15 and $0.10 \mathrm{~V} \mathrm{dec}{ }^{-1}$, and high mobilities of 0.56 and $2.73 \mathrm{~cm}^{2} \mathrm{~V}^{-1} \mathrm{~s}^{-1}$, respectively. More importantly, the low defect density of 1 per $9.22 \times 10^{3}$ and $1.82 \times 10^{4}$ molecules, respectively, confirmed their high crystal quality.
\end{abstract}

Organic Field-effect transistors (OFETs) featuring flexibility and switch function ${ }^{1,2}$ are very important for next-generation logical circuits. Though there are many studies on OFETs demonstrating higher mobility than polycrystalline silicon, the hysteresis and subthreshold swing $(S),{ }^{3,4}$ which determine the response speed of transistors, are still significantly larger than those of inorganic transistors (Table S6, ESI $\dagger$ ). There is still a lack of systematic studies and efficient methods to reduce the $S$ of OFETs. High trap density in organic semiconductors limits the further reduction of $S$ and improves their corresponding response speed. ${ }^{5}$ Organic crystals featuring low impurity and clear structural information are convenient for the quantitative study of the structure-property relationship and development of high-performance devices. ${ }^{6-8}$ OFETs demand lamellar crystals for efficient carrier injections, ${ }^{9-11}$ whereas the shapes of most organic crystals are bulk and very difficult to control.

Herein, we report two organic lamellar crystals, shapes of which guaranteed enough electric field for efficient charge injection, featuring low trap density (TD) in the crystal, fast

State Key Laboratory of Luminescent Materials and Devices, Institute of Polymer Optoelectronic Materials and Devices, South China University of Technology,

No. 381 Wushan Road, Tianhe, Guangzhou 510640, P. R. China.

E-mail: gucheng@scut.edu.cn,ygma@scut.edu.cn

$\dagger$ Electronic supplementary information (ESI) available: Synthetic process, ${ }^{1} \mathrm{H}$-NMR, ${ }^{13} \mathrm{C}-\mathrm{NMR}$, mass spectra, TGA, DSC, PVT growth parameters, crystal structure, cyclic voltammetry, device fabricating, optical properties, tables, and crystal structures. CCDC 2032334 and 1936361. For ESI and crystallographic data in CIF or other electronic format see DOI: 10.1039/d0cc06552h

$\$$ The authors contributed equally. response speed and hysteresis-free OFETs. The crystals were constructed by combining $\pi-\pi$ interactions and intermolecular hydrogen bonds in orthogonal directions, whereas there were only weak van der Waals forces in the third direction. The stronger interactions dominated the growth directions during the crystallisation and resulted in lamellar crystals, which was verified in our previous studies. OFETs based on the lamellar crystals were fabricated, then their device performances were optimised by the modification of the dielectric layer, resulting in high-performance transistors with mobilities up to 0.56 and $2.73 \mathrm{~cm}^{2} \mathrm{~V}^{-1} \mathrm{~s}^{-1}$ for the two crystals, respectively. The $I-V$ curves were carefully analysed, and the resulting trap densities of less than $1.02 \times 10^{10}$ and $4.64 \times 10^{10} \mathrm{~cm}^{-2}$ quantificationally showed high quality of our two organic crystals, respectively.

The cyano-substituted oligo( $p$-phenylene vinylene) (CN-OPV) derivatives, namely $\left(2 Z, 2^{\prime} Z\right)-2,2^{\prime}$-(1,4-phenylene)bis(3-(thiophen-3-yl)acrylonitrile) ( $\alpha$-PBTA) and $\left(2 Z, 2^{\prime} Z\right)-3,3^{\prime}$-(benzo[1,2-b:5,4- $\left.b^{\prime}\right]$ dithiophene-2,6-diyl)bis(2-(thiophen-3-yl)acrylonitrile) ( $\beta$-DBPA) were synthesised by Knoevenagel reaction ${ }^{12}$ (ESI, $\dagger$ and Fig. S1-S10). Their high-quality lamellar crystal forms were obtained by carefully optimising growth conditions during the physical vapor transport (PVT) method $^{13}$ (Fig. S11 and Tables S1, S2, ESI $\dagger$ ), resulting in crystals with sizes up to several hundreds of micrometres and thickness no more than several hundreds of nanometres. The crystals showed no boundaries and cracks under UV irradiation, appearing to be of very high quality. The crystal structures were obtained from single crystal XRD experiments. The crystals of $\alpha$-PBTA showed a triclinic lattice with the space group $P \overline{1}$ and cell parameters of $a=3.9383(8) \AA, b=5.8698(12) \AA, c=18.065(4) \AA$, $\alpha=80.840(3), \beta=87.250(3)^{\circ}, \gamma=86.08(3)^{\circ}$ (Fig. S12a, $b$ and Table S2, ESI $\dagger$ ). The molecules stack into lattice columns through $\pi-\pi$ interaction along the [110] direction one on top of the other with the slip angle of $84.90^{\circ}$ along the long molecule axis (Fig. 1b), and the distance between the adjoining $\pi$-plane is $3.499 \AA$. The multiple hydrogen bonds interconnect the adjacent molecules with each other within the molecular plane to form the layer-like molecule packing (Fig. 1a). Furthermore, the layer-by-layer structure with 


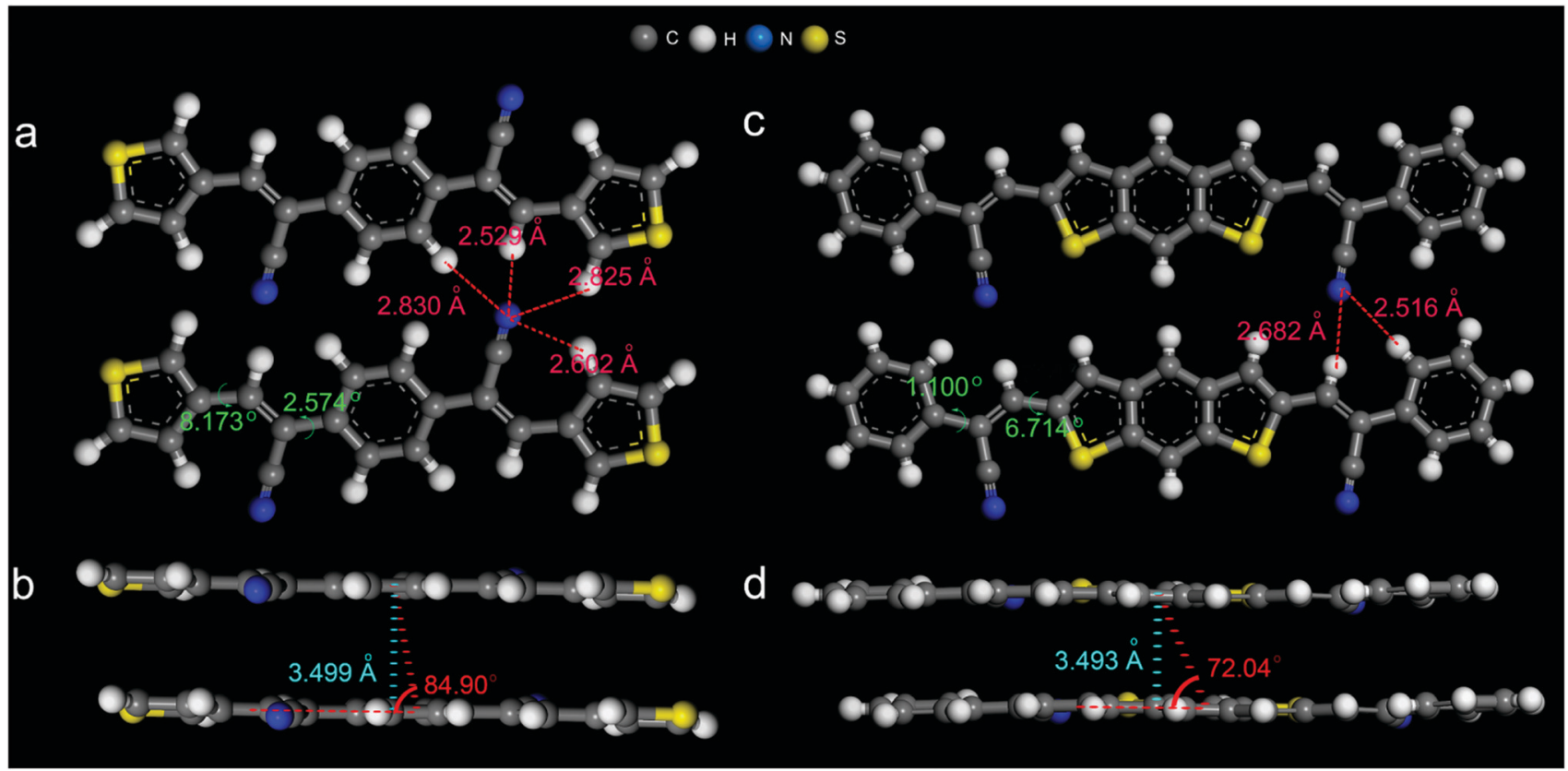

Fig. 1 (a) The length of the hydrogen bonds (red color) and the molecular twisted angle (green color) in the crystal of $\alpha$-PBTA. (b) The $\pi-\pi$ distance (red color) and the slipping angle (sky-blue color) in the crystal of $\alpha$-PBTA. (c) The length of the hydrogen bonds (red color) and the molecular twisted angle (green color) in the crystal of $\beta$-DBPA. (d) The $\pi-\pi$ distance (red color) and the slipping angle (sky-blue color) in the crystal of $\beta$-DBPA.

uniaxially orientated stacking between adjacent layers formed, as shown in Fig. 1b. The molecules showed a very small twisted angle along with its molecular skeleton $\left(8.173^{\circ}\right.$ and $2.574^{\circ}$, Fig. 1c). The crystal density was $1.392 \mathrm{~g} \mathrm{~cm}^{-3}$. On the other hand, the crystals of $\beta$-DBPA showed a monoclinic lattice with the space group of $P 2_{1}$ and cell parameters of $a=3.9035(4) \AA, b=44.025(6) \AA, c=6.0394(6) \AA$, $\alpha=\gamma=90^{\circ}, \beta=95.691^{\circ}$ (Fig. S12c, $d$ and Table S2, ESI $\dagger$ ). The molecules stack into lattice columns through the $\pi-\pi$ interaction along an $a$-axis one on top of the other with the slip angle of $72.04^{\circ}$ along the long molecule axis (Fig. 1d), and the distance between the adjoining $\pi$-plane is $3.493 \AA$. The multiple hydrogen bonds interconnect the adjacent molecules with each other along the [101] direction to form the layer-like molecular packing (Fig. 1c). Furthermore, a layer-by-layer structure with herringbone stacking between the adjacent layers formed, as shown in Fig. S12d (ESI $\dagger$ ). The molecules showed a very small twisted angle along with its molecular skeleton $\left(1.100^{\circ}\right.$ and $6.714^{\circ}$, Fig. 1c). The closer molecular stacking resulted in a higher crystal density of $1.430 \mathrm{~g} \mathrm{~cm}^{-3}$, together with the typical H-type aggregation and the smaller twisted skeleton were superior to charge migrations, which was compatible with the fabrication of high-performance OFETs.

With the obtained lamellar crystals, OFETs were fabricated with $\mathrm{PMMA}^{14}$ as the initial buffer layer on the silicon dioxide $\left(\mathrm{SiO}_{2}\right)$ dielectric layer since it is the most commonly utilised and usually brings considerable performance. All the OFETs were applied bottom gate/top contact (BG/TC) configuration to promote crystal/electrode contacts. At the very beginning, calcium and gold were respectively employed as the source and drain electrodes to form asymmetric OFETs (Fig. S17a, ESI $\dagger$ ) and evaluated if there were any ambipolar characteristics. It turned out that all the transistors demonstrated only typical
P-type unipolar $I-V$ curves. Then, symmetric OFETs with gold electrodes (Fig. S13b, ESI $\dagger$ ) were fabricated. The $I-V$ curves of OFETs based on $\alpha$-PBTA exhibited an obvious nonlinear increase in the initial part of the output curves (Fig. 2a, dashed lines), which was a typical feature of the Schottky contact with obvious injection barriers. ${ }^{5}$ When the applied gate voltage $\left(V_{\mathrm{g}}\right)$ was $-80 \mathrm{~V}$, the maximum current density $\left(J_{\text {out,max }}\right)$ of $0.201 \mathrm{kA} \mathrm{cm}^{-2}$ was estimated with the measured maximum output drain current $\left(I_{\mathrm{d}}\right)$ of $1.73 \mu \mathrm{A}$, and the channel width of $850 \mu \mathrm{m}$ as well as the channel thickness of $1 \mathrm{~nm}$. The carrier mobility of $0.11 \mathrm{~cm}^{2} \mathrm{~V}^{-1} \mathrm{~s}^{-1}$ and the threshold voltage of $-28.77 \mathrm{~V}$ were extracted from the saturation region of transfer curves (Fig. 2b, dash line). Its minimum $S$ in transfer curves was $1.5 \mathrm{~V} \mathrm{dec}^{-1}$, from which the TD of $1.81 \times 10^{12} \mathrm{~cm}^{-2}$ in the crystal/dielectric buffer layer interface was extracted according to $N_{\mathrm{t}}=[S \log (e) /$ $(k T / q)-1] C_{\mathrm{i}} / q,{ }^{11}$ and the method is described in detail in the ESI. $\dagger$ Moreover, the $I-V$ curves of OFETs based on the lamellar crystal of $\beta$-DBPA also exhibit an obvious nonlinear increase in the initial part of output curves (Fig. 2c, dashed lines). The $I_{\mathrm{d}}$ was kept less than $1 \mathrm{nA}$ until the drain voltage $\left(V_{\mathrm{d}}\right)$ was higher than $-10 \mathrm{~V}$. The maximum $I_{\mathrm{d}}$ was $7.05 \mu \mathrm{A}$ when the $V_{\mathrm{g}}$ was only $-60 \mathrm{~V}$. Its $J_{\text {out,max }}$ reached $11.2 \mathrm{kA} \mathrm{cm}^{-2}$ according to the channel width of $63.3 \mu \mathrm{m}$. The carrier mobility of $1.71 \mathrm{~cm}^{2} \mathrm{~V}^{-1} \mathrm{~s}^{-1}$ and the threshold voltage of $-20.83 \mathrm{~V}$ were calculated from the saturation region of transfer curves (Fig. 2d, dashed lines). The obtained minimum $S$ was $0.75 \mathrm{~V} \mathrm{dec}^{-1}$, by which the minimum defect density in the interface of $8.69 \times$ $10^{11} \mathrm{~cm}^{-2}$ was estimated. A smaller hysteresis of the transfer curve was observed in the OFETs when $V_{\mathrm{g}}$ was swept forward and backward, with the on/off ratio ( $\left.I_{\text {on/off }}\right)$ higher than $10^{6}$, as illustrated in detail in Table S4 (ESI $\dagger$ ). 

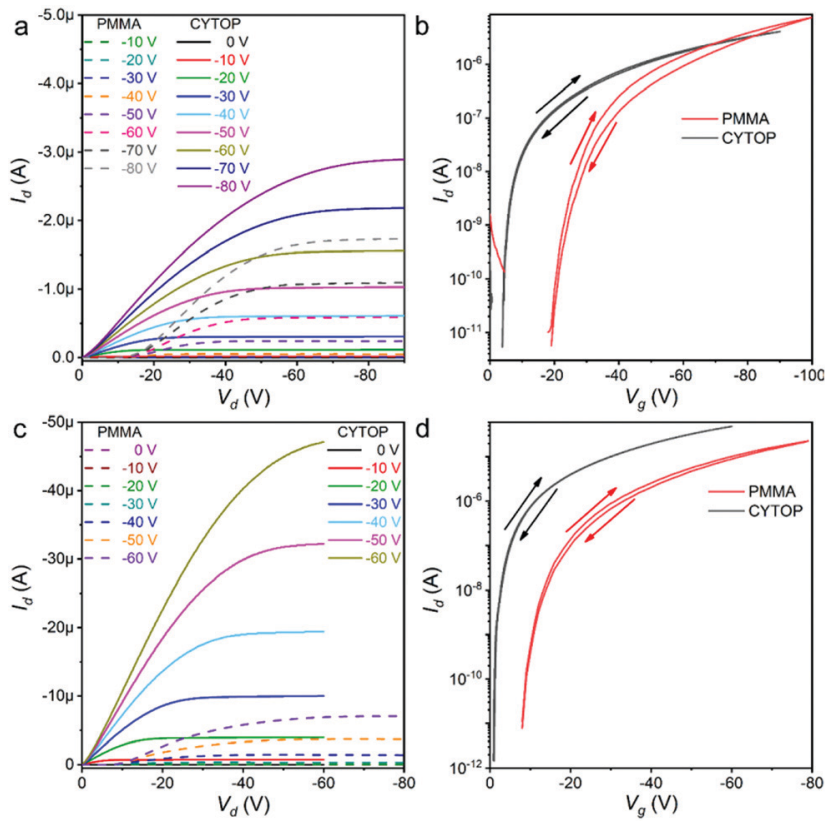

Fig. 2 (a) Output curves of OFETs based on the single crystal of $\alpha$-PBTA. The dashed curves were the $I-V$ curves from OFETs with the PMMA buffer layer, while the solid curves were the I-V curves from OFETs with the CYTOP buffer layer. (b) Transfer characteristics of the transistors based on the single crystals of $\alpha$-PBTA. In the transfer characteristics, $V_{d}=-110$ and $-80 \mathrm{~V}$ for OFETs with (red line) PMMA and (black line) CYTOP buffer layers, respectively. (c) Output curves of OFETs are based on the single crystal of $\beta$-DBPA. The dash curves were the $I-V$ curves from OFETs with the PMMA buffer layer, while the solid curves were the $I-V$ curves from OFETs with the CYTOP buffer layer. (d) Transfer characteristics of the transistors based on the single crystals of $\beta$-DBPA. In the transfer characteristics, $V_{d}=-80$ and $-60 \mathrm{~V}$ for OFETs with (red line) PMMA and (black line) CYTOP buffer layers, respectively. The transfer curves were swept forward and backward to evaluate their hysteresis.

To reduce the injection barrier and improve the device performance, perfluoro(1-butenylvinylether)polymer (CYTOP) ${ }^{15}$ was utilised as a buffer layer instead of PMMA (Fig. S17c, ESI†).
The devices were fabricated under otherwise identical conditions. OFETs based on two lamellar crystals demonstrated much superior performances. In the transistors of $\alpha$-PBTA, the drain current $\left(I_{\mathrm{d}}\right)$ almost linearly increased along with the increase in $V_{\mathrm{d}}$ (Fig. 2a, solid lines), indicating the resistance between the crystal and gold electrodes was almost negligible. The $J_{\text {out,max }}$ of $7.61 \mathrm{kA} \mathrm{cm}^{-2}$ was estimated when $V_{\mathrm{g}}=-60 \mathrm{~V}$ with the measured maximum $I_{\mathrm{d}}(2.89 \mu \mathrm{A})$ and the channel width of $38.0 \mu \mathrm{m}$. The minimum $S$ in the transfer curves decreased to $0.15 \mathrm{~V} \mathrm{dec}^{-1}$ (Fig. 2b, solid lines), which was reduced ten-folds compared with that in the OFETs with PMMA as the buffer layer. The extracted defect density in the interface was only $1.02 \times 10^{11} \mathrm{~cm}^{-2}$. The hysteresis of transfer curves was only $0.8 \mathrm{~V}$ when $V_{\mathrm{g}}$ was swept forward and backward. The improvements in the transistors of $\beta$-DBPA were quite similar. In the output curves (Fig. 2c, solid lines), the $I_{\mathrm{d}}$ increased linearly with the $V_{\mathrm{d}}$ in the initiation region, and the intercept between the $Y$-axis and $I_{\mathrm{d}}$ of the linear region came across the original point, indicating that the interface of the crystal and electrodes became Ohmic contact. The output current at a high voltage was limited by the space-chargelimited current (SCLC). The maximum $I_{\mathrm{d}}$ in the transistors reached $47.3 \mu \mathrm{A}$ when the $V_{\mathrm{g}}$ was only $-60 \mathrm{~V}$, and the $J_{\text {out,max }}$ was $58.5 \mathrm{kA} \mathrm{cm}^{-2}$ in the device with a channel width of $80.9 \mu \mathrm{m}$. The resulting maximum current density was 4.22 times higher than that in the transistors with the PMMA buffer layer under the same $V_{g}$ (Table S4, ESI $\dagger$ ). From the transfer curves (Fig. 2d, solid lines), the threshold voltage decreased to $-3.60 \mathrm{~V}$. The minimum $S$ decreased from $0.75 \mathrm{~V} \mathrm{dec}^{-1}$ to $0.10 \mathrm{~V} \mathrm{dec}^{-1}$. The extracted TD in the interface was $4.64 \times 10^{10} \mathrm{~cm}^{-2}$, which was one of the transistors with the lowest $S$ and TD (Table 1), as obtained by OFETs. With the lattice parameters, we could quantitatively know the quality of the crystals; the calculation method is listed in the ESI, $\uparrow$ (calculation of defects in organic crystals) and eqn (S4) and (S5) (ESI $\dagger$ ). The molecule densities of $\alpha$-PBTA and $\beta$-DBPA in the carrier migration plane were

Table $1 S$ and TD in amorphous and single crystal-based OFETs

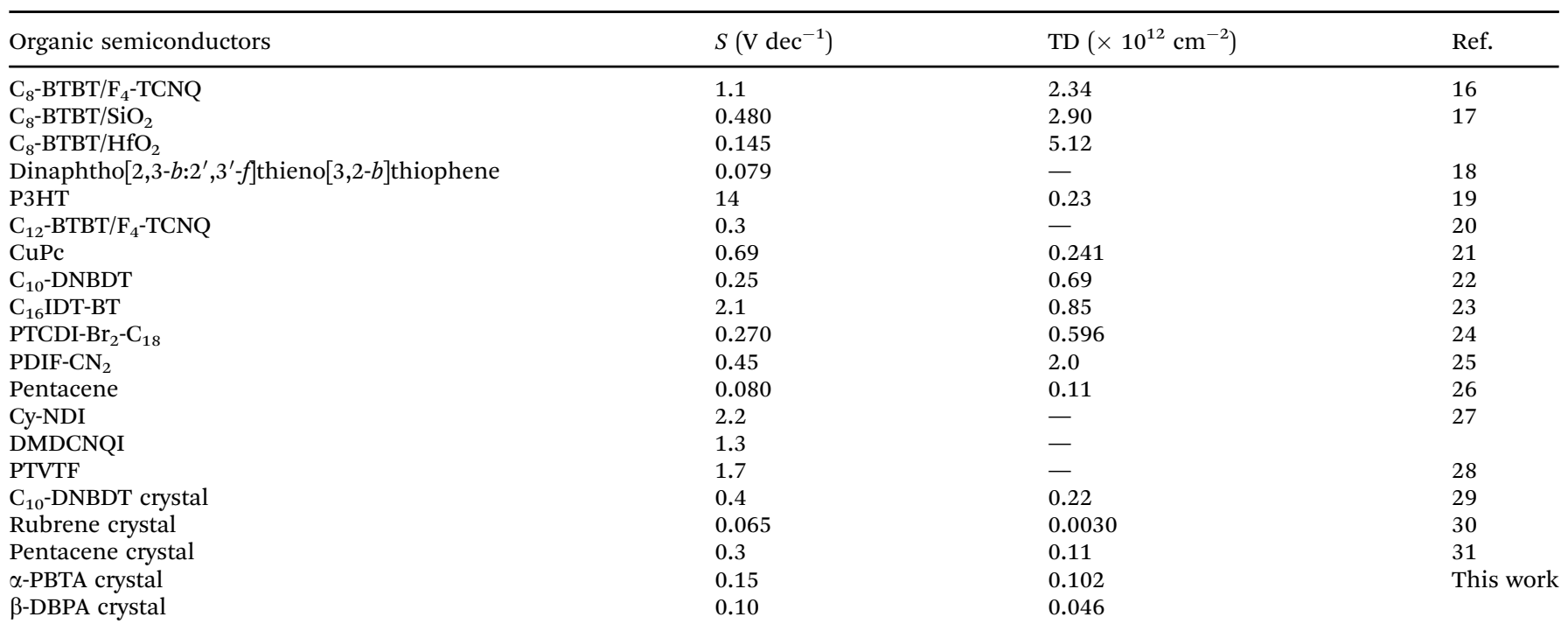

- Not mentioned, and could not be calculated due to lack of necessary data. 
$9.40 \times 10^{14}$ and $8.43 \times 10^{14} \mathrm{~cm}^{-2}$, respectively. Thus, the resulting deep-TD was less than 1 per $9.22 \times 10^{3}$ and $1.82 \times 10^{4}$ molecules in the crystal of $\alpha$-PBTA and $\beta$-DBPA, respectively. To the best of our knowledge, only rubrene crystals with a careful treatment achieved better quality, ${ }^{30}$ indicating the outstanding quality of our crystals. The carrier mobility was derived from the transfer curves in the saturation region, which were dependent on the variation of $V_{g}$, which ensured the accuracy of the calculated carrier mobility. The highest hole mobilities of $\alpha$-PBTA and $\beta$-DBPA were 0.56 and $2.73 \mathrm{~cm}^{2} \mathrm{~V}^{-1} \mathrm{~s}^{-1}$, respectively (Table $\mathrm{S} 4$, ESI $\dagger$ ). According to the crystal structure analyses, $\beta$-DBPA had a closer and larger $\pi-\pi$ overlap, which increases the overlap integral and improves the carrier mobility. ${ }^{32}$ Moreover, $\beta$-DBPA had stronger interactions between adjacent molecules, which were critical to decrease the TD of crystal $^{33}$ and further improve the device performance.

In conclusion, the integration of multiple intermolecular hydrogen bonds and $\pi-\pi$ interactions during crystal engineering was critical to develop high quality lamellar organic single crystals compatible with the device fabrication. Closer intermolecular packing brings stronger intermolecular forces, reduce the trap density from $1.02 \times 10^{11} \mathrm{~cm}^{-2}$ in the crystal of $\alpha$-PBTA to $4.64 \times 10^{10} \mathrm{~cm}^{-2}$ and in $\beta$-DBPA, improves the carrier mobility from $0.56 \mathrm{~cm}^{2} \mathrm{~V}^{-1} \mathrm{~s}^{-1}$ to $2.73 \mathrm{~cm}^{2} \mathrm{~V}^{-1} \mathrm{~s}^{-1}$, resulting in faster on/ off switch speed in the transistors of $\beta$-DBPA. This work demonstrated a method to study the structure-property relationship quantificationally and an efficient way to obtain high-quality organic crystals for high-performance OFETs.

This work was supported by the National Natural Science Foundation of China (No. 91833304, 21975078 and 51703065), the Introduced Innovative R\&D Team of Guangdong Province (201101C0105067115), the Natural Science Foundation of Guangdong Province (2019B030301003), the 111 Project, the Fundamental Research Funds for the Central Universities, and the Thousand Youth Talents Plan.

\section{Conflicts of interest}

There are no conflicts to declare.

\section{Notes and references}

1 T. Someya, T. Sekitani, S. Iba, Y. Kato, H. Kawaguchi and T. Sakurai, Proc. Natl. Acad. Sci. U. S. A., 2004, 101, 9966-9970.

2 T. Sekitani, S. Iba, Y. Kato, Y. Noguchi, T. Someya and T. Sakurai, Appl. Phys. Lett., 2005, 87, 173502.

3 K. P. Pernstich, S. Haas, D. Oberhoff, C. Goldmann, D. J. Gundlach, B. Batlogg, A. N. Rashid and G. Schitter, J. Appl. Phys., 2004, 96, 6431-6438.
4 J. K. Jeong, J. H. Jeong, H. W. Yang, J.-S. Park, Y.-G. Mo and H. D. Kim, Appl. Phys. Lett., 2007, 91, 113505.

5 G. Horowitz, Adv. Mater., 1998, 10, 365-377.

6 H. M. Chen, X. Xing, M. A. Zhu, J. P. Cao, M. U. Ali, A. Y. Li, Y. W. He and H. Meng, ACS Appl. Mater. Interfaces, 2019, 11, 34188-34195.

7 M. Mas-Torrent and C. Rovira, Chem. Rev., 2011, 111, 4833-4856.

8 F. Di Maria, E. Fabiano, D. Gentili, M. Biasiucci, T. Salzillo, G. Bergamini, M. Gazzano, A. Zanelli, A. Brillante, M. Cavallini, F. Della Sala, G. Gigli and G. Barbarella, Adv. Funct. Mater., 2014, 24, 4943-4951.

9 F. Yakuphanoglu and B. F. Şenkal, Polym. Eng. Sci., 2009, 49, 722-726.

10 F. Yakuphanoglu, C. Soykan and R. Coşkun, Polym. Int., 2008, 57, 324-328.

11 F. Yakuphanoglu, Phys. B, 2007, 393, 139-142.

12 C. Simon, T. Constantieux and J. Rodriguez, Eur. J. Org. Chem., 2004, 4957-4980.

13 C. Kloc, P. G. Simpkins, T. Siegrist and R. A. Laudise, J. Cryst. Growth, 1997, 182, 416-427.

14 G. Horowitz, Adv. Mater., 1996, 8, 177-179.

15 S. Haas, Y. Takahashi, K. Takimiya and T. Hasegawa, Appl. Phys. Lett., 2009, 95, 022111.

16 K. Pei, A. H. Y. Lau and P. K. L. Chan, Phys. Chem. Chem. Phys., 2020, 22, 7100-7109.

17 S. Jiang, Q. Wang, J. Qian, J. Guo, Y. Duan, H. Wang, Y. Shi and Y. Li, ACS Appl. Mater. Interfaces, 2020, 12, 26267-26275.

18 M. Geiger, R. Acharya, E. Reutter, T. Ferschke, U. Zschieschang, J. Weis, J. Pflaum, H. Klauk and R. T. Weitz, Adv. Mater. Interfaces, 2020, 7, 1902145.

19 F. Zhang, Y. F. Hu, Z. D. Lou, X. G. Xin, M. Zhang, Y. B. Hou and F. Teng, RSC Adv., 2018, 8, 11272-11279.

20 P. Wei, Y. P. Hu, Y. W. Zhu, Y. H. Jiang, X. S. Feng, S. T. Li, L. J. Bu, X. N. Yang and G. H. Lu, Adv. Electron. Mater., 2018, 4, 1800339.

21 L. Vijayan, A. Thomas, K. S. Kumar and K. B. Jinesh, J. Sci.: Adv. Mater. Devices, 2018, 3, 348-352.

22 T. Makita, M. Sasaki, T. Annaka, M. Sasaki, H. Matsui, C. Mitsui, S. Kumagai, S. Watanabe, T. Hayakawa, T. Okamoto and J. Takeya, Appl. Phys. Lett., 2017, 110, 163302.

23 W. Tang, J. Q. Zhao, L. R. Feng, P. F. Yu, W. M. Zhang and X. J. Guo, IEEE Electron Device Lett., 2015, 36, 59-61.

24 N. V. V. Subbarao, M. Gedda, S. Vasimalla, P. K. Iyer and D. K. Goswami, Phys. Status Solidi A, 2014, 211, 2403-2411.

25 K. Willa, R. Hausermann, T. Mathis, A. Facchetti, Z. Chen and B. Batlogg, J. Appl. Phys., 2013, 113, 133707.

26 A. Petritz, A. Wolfberger, A. Fian, J. R. Krenn, T. Griesser and B. Stadlober, Org. Electron., 2013, 14, 3070-3082.

27 J. M. Cho, Y. Akiyama, T. Kakinuma and T. Mori, AIP Adv., 2013, $3,102131$.

28 D. S. Chung, S. J. Lee, J. W. Park, D. B. Choi, D. H. Lee, J. W. Park, S. C. Shin, Y. H. Kim, S. K. Kwon and C. E. Park, Chem. Mater., 2008, 20, 3450-3456.

29 S. Sakai, J. Soeda, R. Hausermann, H. Matsui, C. Mitsui, T. Okamoto, M. Ito, K. Hirose, T. Sekiguchi, T. Abe, M. Uno and J. Takeya, Org. Electron., 2015, 22, 1-4.

30 B. Blulle, R. Hausermann and B. Batlogg, Phys. Rev. Appl., 2014, 1, 034006.

31 W. L. Kalb and B. Batlogg, Phys. Rev. B: Condens. Matter Mater. Phys., 2010, 81, 035327.

32 R. A. Marcus, J. Chem. Phys., 1956, 24, 966-978.

33 T. Soma and A. Morita, J. Phys. Soc. Jpn., 1972, 32, 357-364. 